\title{
A Case of Diabetic Macular Edema with Prominent Chorioretinal Folds
}

\author{
Takaki Sato $^{\mathrm{a}}$ Ryohsuke Kohmoto $^{\mathrm{a}}$ Masanori Fukumoto $^{\mathrm{a}}$ \\ Seita Morishita ${ }^{a, b}$ Daisaku Kimura ${ }^{a, c}$ Kensuke Tajiri ${ }^{a}$ \\ Takatoshi Kobayashi $^{a}$ Teruyo Kida ${ }^{a}$ Shota Kojima ${ }^{a}$ Tsunehiko Ikeda ${ }^{a}$ \\ ${ }^{a}$ Department of Ophthalmology, Osaka Medical College, Takatsuki, Japan; ${ }^{b}$ Department of \\ Ophthalmology, Osaka Kaisei Hospital, Takatsuki, Japan; 'Department of Ophthalmology, \\ Takatsuki Red Cross Hospital, Takatsuki, Japan
}

\section{Keywords}

Diabetic retinopathy $\cdot$ Cystoid macular edema $\cdot$ Chorioretinal fold · Vitreous surgery

Hypertension · Diabetic nephropathy

\begin{abstract}
Purpose: To report a case of diabetic macular edema with prominent chorioretinal folds. Case Report: This study involved a 55-year-old male with untreated bilateral diabetic retinopathy who had undergone cataract surgery at another clinic. Following that surgery, diabetic macular edema rapidly exacerbated, accentuating marked cystoid macular edema and radial chorioretinal folds in the macula. Investigation of his medical history revealed that in addition to diabetes, he had uncontrolled hypertension and severe diabetic nephropathy. Vitreous surgery was performed on both eyes due to a resistance to a subtenon injection of triamcinolone acetonide or intravitreal injection of an antivascular endothelial growth factor agent. After surgery, the macular edema and chorioretinal folds showed a tendency towards improvement. Thereafter, kidney transplant surgery was performed for renal failure, and a mild tendency of chorioretinal folds was observed. Conclusion: In the case presented in this study, we observed remarkable cystoid macular edema in the fovea centralis and theorize that distortion with the surrounding tissue might have occurred, thus leading to the formation of chorioretinal folds around the macula.




\section{Introduction}

Chorioretinal folds are known to occur due to a variety of factors, such as low ocular pressure, scleral buckling surgery, orbital tumor, and thyroid eye disease [1-7]. However, it is rare for prominent chorioretinal folds to occur concurrently with diabetic macular edema. In the present study, we report a case of diabetic macular edema that was accompanied by prominent chorioretinal folds.

\section{Case Report}

This study involved a 55-year-old male who had previously been diagnosed with diabetes mellitus, and who had untreated bilateral diabetic retinopathy. Diabetic macular edema rapidly exacerbated after he underwent cataract surgery in both eyes at another clinic. Investigation of his medical history revealed that in addition to diabetes, he had reduced renal function (creatinine $426 \mathrm{U} / \mathrm{L}$, eGFR 24.5 (30> eGFR $\geq 15$ ) due to poorly controlled high blood pressure and diabetic nephropathy. However, his thyroid function was normal (free T4 1.21 $\mathrm{ng} / \mathrm{dL}, \mathrm{TSH} 4.66 \mu \mathrm{U} / \mathrm{mL})$.

\section{Clinical Findings at Initial Examination}

Upon initial examination, the patient's visual acuity was 0.1 (uncorrectable) OD and 0.1 $(0.3 \mathrm{p} \times \mathrm{S}+1.50 \mathrm{dpt}) \mathrm{OS}$, and his intraocular pressure was $14 \mathrm{~mm} \mathrm{Hg}$ OD and $15 \mathrm{~mm} \mathrm{Hg}$ OS. The axial length was $22.5 \mathrm{~mm}$ OD and $22.7 \mathrm{~mm}$ OS. Retinal hemorrhage and numerous soft exudates were observed in the wide range of the fundus, thus showing the stage of preproliferative diabetic retinopathy (Fig. 1a, b). Examination by optical coherence tomography revealed diffuse macular edema and advanced cystoid macular edema in the fovea (Fig. 1c, d). In addition, radial chorioretinal folds were observed in the macula.

\section{Clinical Course After Initial Examination}

Injection of triamcinolone acetonide into the sub-Tenon's capsule and injection of an antivascular endothelial growth factor agent resulted in no improvement of the macular edema, so we subsequently performed vitreous surgery on both eyes. Three weeks after surgery, the macular edema and chorioretinal folds showed a tendency of improvement. At the patient's follow-up visit 1.5 years after vitreous surgery, we found that his vision had improved, and that his visual acuity was $0.7 \times \mathrm{S}+1.25 \mathrm{dpt}=\mathrm{C}-0.5 \mathrm{DA} \times 90^{\circ} \mathrm{OD}$ and $0.3 \times \mathrm{S}+$ $1.25 \mathrm{dpt}=\mathrm{C}-0.75 \mathrm{DA} \times 85^{\circ}$ OS and that his intraocular pressure was $19 \mathrm{~mm} \mathrm{Hg}$ OD and 15 $\mathrm{mm} \mathrm{Hg}$ OS. In both eyes, the macular edema and chorioretinal folds showed improvement, though not complete (Fig. 2). After vitreous surgery, the patient underwent kidney transplantation surgery due to the reduction of his renal function. After the kidney transplantation, the macular edema and chorioretinal folds showed a tendency to improve.

\section{Discussion}

Ophthalmologic factors, such as hypotensive pressure [1], uveitis [2], age-related macular degeneration [3], scleral buckling surgery [4], and orbital tumor [5], etc., are known to be causes of chorioretinal folds. In addition, there have been reports of thyroid ophthalmopathy [6], resulting from systemic disease and idiopathic intracranial hypertension [7], etc. How- 
ever, it is rare to observe chorioretinal folds in cases of diabetic macular edema. In a study on the relationship between diabetic retinopathy and chorioretinal folds, Fagúndez Vargas et al. [8] reported that choroid folds were observed in 16 out of 1,019 cases of diabetic retinopathy. Moreover, they reported that the respective risk factors consisted of: (1) type 2 diabetes $(100 \%$ of the cases), (2) female gender ( $88 \%$ of the cases), (3) insulin use dependence ( $85 \%$ of the cases), and (4) mild hypermetropia (i.e., less than $2.5 \mathrm{dpt}$; $54 \%$ of the cases). In addition, moderate or higher-level diabetic retinopathy (i.e., proliferative in $12 \%$ of the cases) was the risk factor in $44 \%$ of the cases, and the general complication of high blood pressure was a risk factor in $50 \%$ of the cases.

In the case presented in our study, bilateral diabetic retinopathy before proliferation and hypertension of poor control was observed, thus illustrating that our patient had type 2 diabetes, which is consistent with the risk factor findings in the above-mentioned study. However, what was inconsistent with the risk factor was the fact that his diabetes mellitus was being controlled by oral treatment, and that the refraction prior to his cataract surgery was myopia. Another characteristic finding in the general condition of this case was that having observed severe diabetic nephropathy, kidney transplantation was performed after vitreous surgery. Thus, it appears that it easily caused advanced macular edema.

In a study of chorioretinal folds that were caused by various factors, Timothy et al. [9] theorized that secondary chorioretinal folds might have been caused by abnormal adhesion of the retina and choroid. In this case, exudative retinal detachment was hardly observed, regardless of high exudation as a form of exudative change. Although the cause was unknown, it seems that there was a possibility that retinal detachment did not occur, despite high exudation due to abnormal adhesion of the retina and choroid. In addition, in a study on chorioretinal folds due to intracranial pressure increase, Sibony et al. [10] reported a mechanism for producing chorioretinal folds in the peripapilla. They speculated that in the case in which papilledema was observed, when the tissues within the papilla expanded, wrinkles might have occurred due to irregular tensile stress to the surrounding tissues in the circumferential direction.

The macular edema in our present case was characterized in form by a flat diffuse macular edema in the para-fovea, despite the fact that a high degree of alveolar macular edema was observed in the fovea. Based on that finding, we theorized that a gap between the fovea and the surrounding tissues was created, thus causing distortion with the surrounding tissues and wrinkles around the macula, i.e. with the same mechanism as the chorioretinal folds occurring around the optic disc at the time of intracranial pressure increase (Fig. 3).

In conclusion, in the case presented here, the improvement of chorioretinal folds was observed after vitrectomy, but in addition to the effect of relieving macular edema by vitreous surgery, the improvement of edema throughout the patient's entire body via kidney transplantation appears to have also contributed to the improvement of his chorioretinal folds.

\section{Acknowledgements}

The authors wish to thank John Bush for editing the manuscript. 


\section{Statement of Ethics}

This case study was approved by the Ethics Committee of Osaka Medical College.

\section{Disclosure Statement}

There are no conflicts of interest to report for all authors.

\section{References}

-1 Fannin LA, Schffman JC, Budenz DL: Risk factors for hypotony maculopathy. Ophthalmology 2003;110:1185-1191.

2 Haruyama M, Yuzawa M, Kawamura A, Yamazaki C, Matsumoto Y: Indocyanine green angiographic findings of chorioretinal folds. Jpn J Ophthalmol 2001;45:293-300.

3 Gass JD: Radial chorioretinal folds. A sign of choroidal neovascularization. Arch Ophthalmol 1981;99:1016-1018.

4 Friberg TR: The etiology of choroidal folds. A biomechanical explanation. Graefes Arch Clin Exp Ophthalmol 1989;227:459-464.

5 Hedges TR Jr, Leopoldo IH: Parallel retinal folds; their significance in orbital space-taking lesions. Arch Ophthalmol 1959;62:353-355.

-6 Kowal L, Georgievski Z: Choroidal folds in Graves' ophthalmopathy. Aust N Z J Ophthalmol 1994;22:216.

-7 Patrick AS, Mark JK, Steven EF, Jun-Kai W, Mora G: Retinal and choroidal folds in papilledema. Invest Ophthalmol Vis Sci 2015;56:5670-5680.

-8 Fagúndez Vargas MA, Jimenez Parras R, Bermudez Uria L: Choroidal folds in diabetic retinopathy. Arch Soc Esp Oftalmol 2000;75:797-802.

-9 Timothy WO, Neal VP, Lyndon BL, Chris SB, Steven Y: Chorioretinal folds: associated disorders and a related maculopathy. Am J Ophthalmol 2014;157:1038-1047.

-10 Sibony PA, Kupersmith MJ, Feldon SE, Wang JK, Garvin M; OCT Substudy Group for the NORDIC Idiopathic Intracranial Hypertension Treatment Trial: Retinal and choroidal folds in papilledema. Invest Ophthalmol Vis Sci 2015;56:5670-5680. 


\section{Case Reports in Ophthalmology}
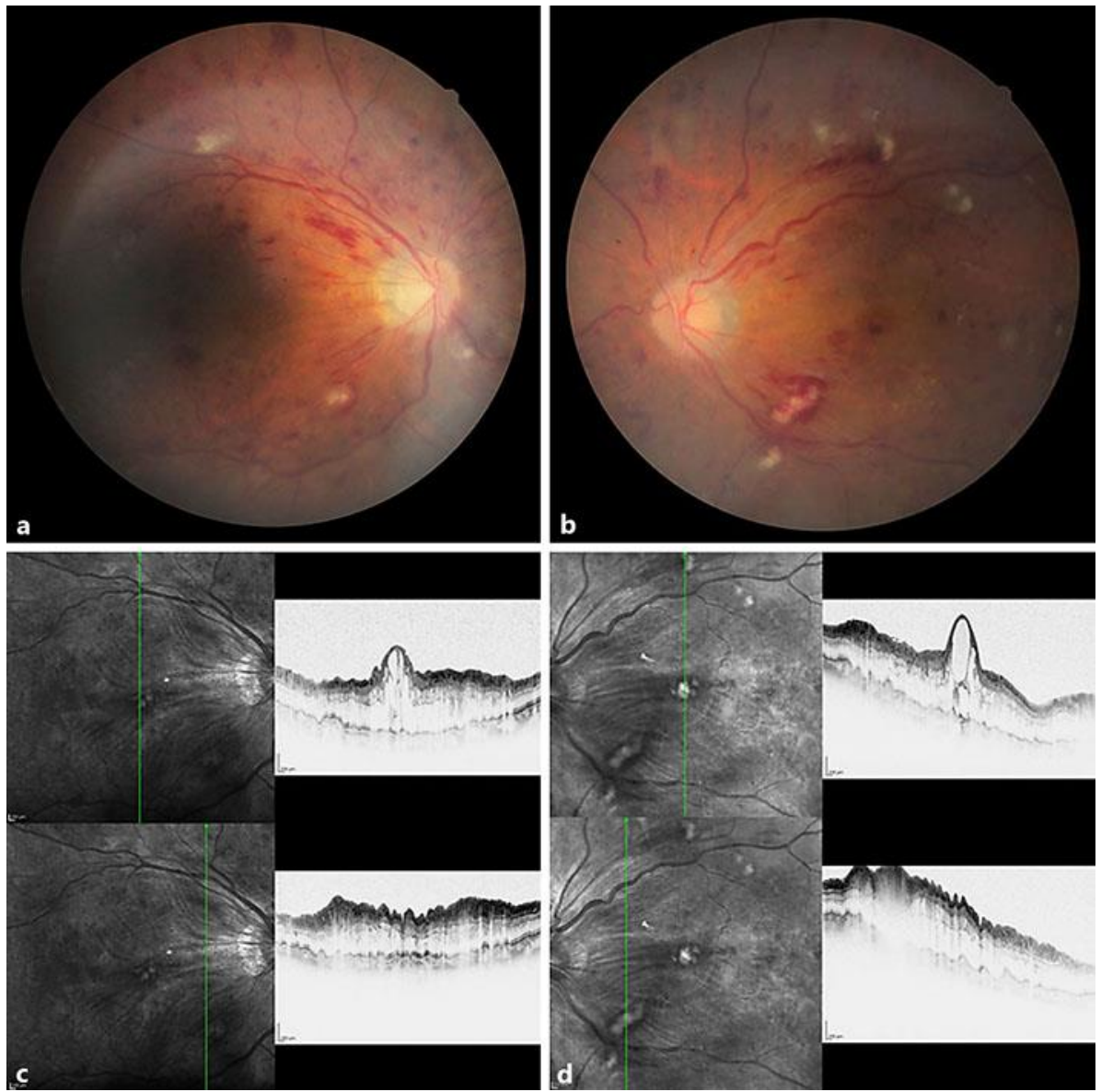

Fig. 1. Fundus photograph and optical coherence tomography (OCT) images obtained at the patient's initial visit. a, b Retinal hemorrhage and numerous soft exudates were observed in the wide range of the fundus, thus showing the stage of preproliferative diabetic retinopathy. $\mathbf{c}, \mathbf{d}$ The OCT images reveal advanced cystoid macular edema and radial chorioretinal folds in the fovea. 


\section{Case Reports in Ophthalmology}

\begin{tabular}{l|l}
\hline Case Rep Ophthalmol 2017;8:163-169 \\
\hline DOI: 10.1159/000461574 & $\begin{array}{l}\text { C 2017 The Author(s). Published by S. Karger AG, Basel } \\
\text { www.karger.com/cop }\end{array}$ \\
\hline
\end{tabular}
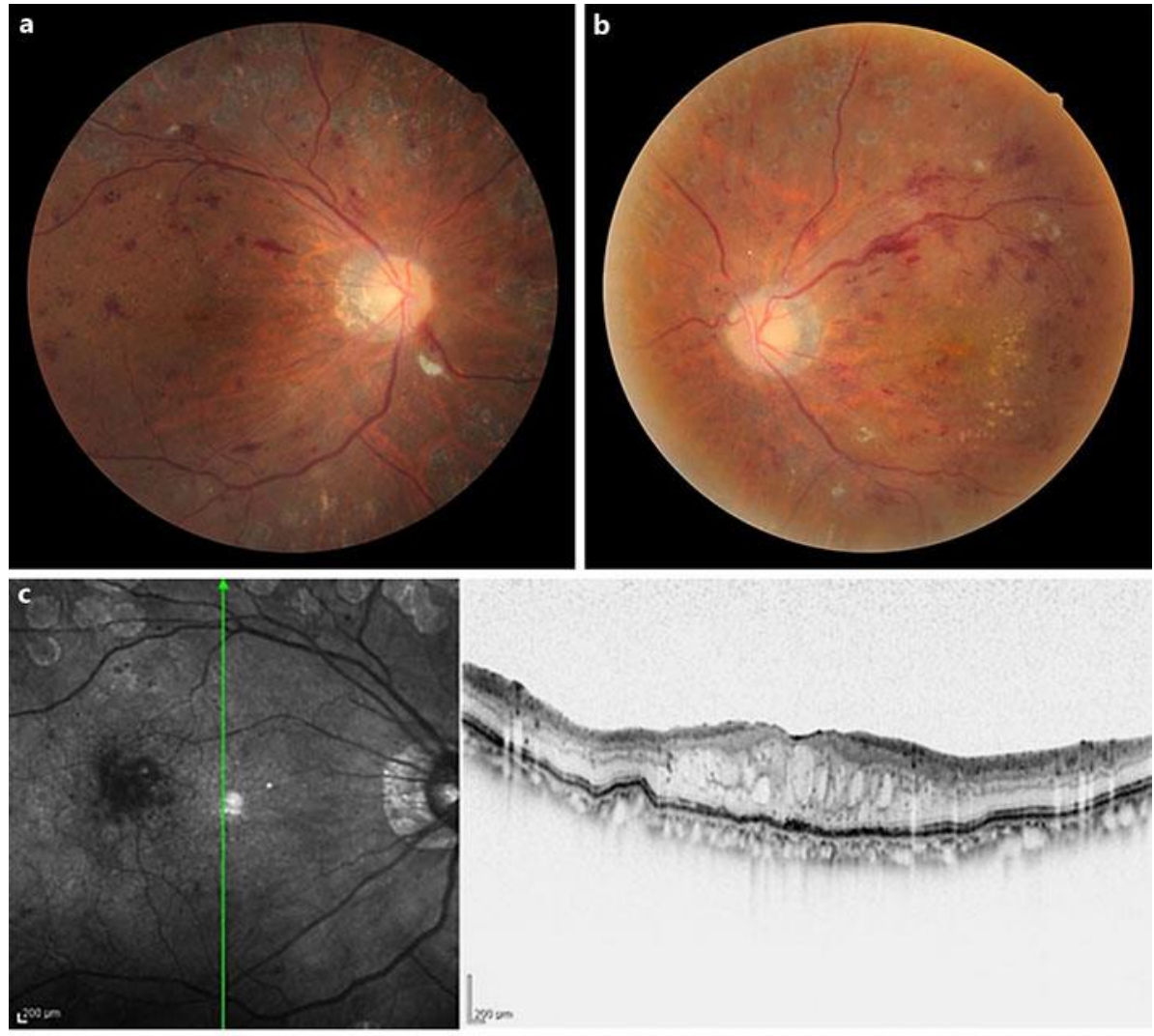

Sato et al.: A Case of Diabetic Macular Edema with Prominent Chorioretinal Folds

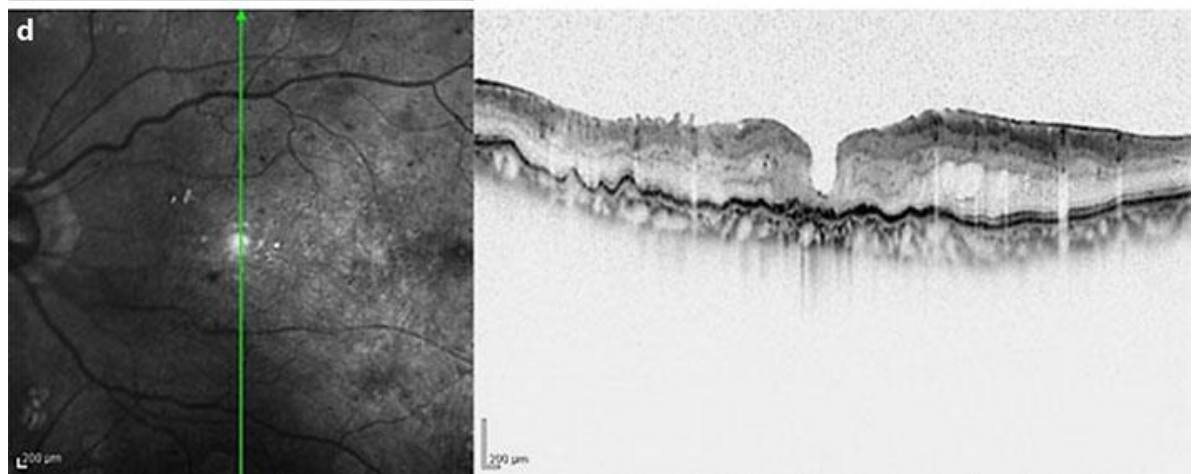

Fig. 2. Fundus photograph and optical coherence tomography (OCT) images obtained at the patient's follow-up visit 1.5 years after vitreous surgery. a, b In both eyes, retinal hemorrhage and soft exudates were slightly decreased. c, d The OCT images revealed improvement of the macular edema and chorioretinal folds, though not complete. 


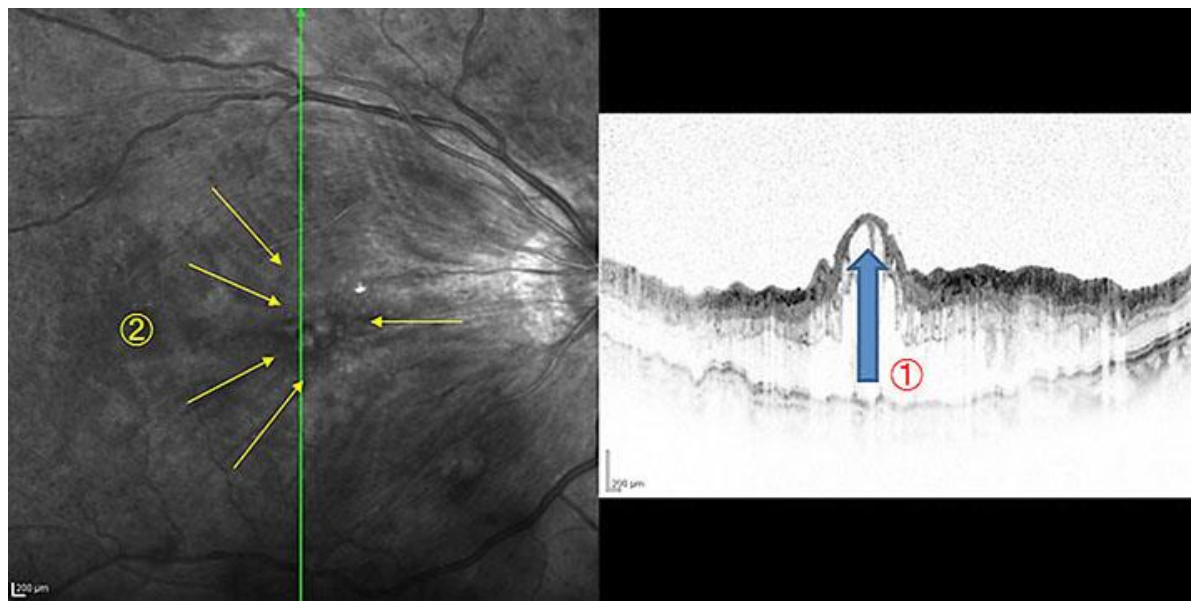

Fig. 3. The mechanism of chorioretinal fold formation. The macular edema in our patient was characterized in form by a flat diffuse macular edema in the para-fovea, despite the fact that a high degree of alveolar macular edema (1) was observed in the fovea. The gap between the fovea and the surrounding tissues was created, thus causing the chorioretinal folds (2). 